\title{
Research progress of active polypeptides of Cordyceps militaris
}

\author{
Guangyu $\mathrm{Xu}^{1}$, Peige $\mathrm{Du}{ }^{1}$, Liping $\mathrm{An}{ }^{1}$, Gang Lv ${ }^{1}$, Lifeng Miao ${ }^{2}$ and Yuan Guangxin ${ }^{1, a}$ \\ ${ }^{1}$ College of Pharmacy, Beihua University, Jilin, Jilin, China \\ ${ }^{2}$ First Clinical Hospital, Beihua University, Jilin, Jilin, China
}

\begin{abstract}
It is recognized at home and abroad that Cordyceps militaris is a fungus that can be edible and used for medicinal application, and also a common cordyceps taishanensis that is widely distributed and with very high medicinal value in China. Active Cordyceps militaris polypeptide has shown several highlights in its absorption mechanism, such as directly absorbed without the need for digestion, and absorbed quickly and completely, and more and more international researchers are attaching great importance to it. Physiological and pharmacological activities of Cordyceps militaris polypeptide are mainly to activate related enzyme systems, promote intermediary metabolisms or control the DNA transcription and translation, simultaneously activate the reticuloendothelial system and macrophage, promote the transformation of lymphocytes, and as a nonspecific immune enhancer and regulator, activate the immune competent cells, especially lymphocytes, lymphokines, mononuclear macrophage system and NK cells, thereby attacking the target cells to play an antitumor role, and also exerting its anti-aging, anticoagulant, hypolipidemic to enhance the immunity, improve the liver function and delay the aging. In this paper, the immunity improvement, anticancer, antioxidation and antimicrobial activities of active polypeptides from Cordyceps militaris are reviewed, in order to provide a solid theoretical help for the further development and application of active polypeptides of Cordyceps militaris.
\end{abstract}

\section{Introduction}

North Cordyceps sinensis, also known as Cordyceps or Cordyceps militaris, and Boschniakia rossica in its popular name, and belongs to the medicinal fungus as does Cordyceps sinensis, is recognized as a type of medicinal fungi that are traditional Chinese health foods at home and abroad, can replace Cordyceps sinensis as a medicine commonly used in China, with some advantages such as high medicinal values, wide distribution, and easiness to be cultivated $[1,2]$. The main components of Cordyceps militaris are cordycepin, polysaccharides and active polypeptides. Due to a few highlights of the active polypeptides in its absorption mechanism, it is attracting more and more researchers' attention. Active polypeptides can be directly absorbed without the need for digestion, and the

\footnotetext{
${ }^{a}$ Corresponding author: yuanguangxin2007@163.com
}

The research supported by Jilin science and technology project $(201464063,20166017)$, Health planning project of Jilin Province (2014Z079), Jilin Province Science and Technology Development Program(2040311031YY, 201603092YY). 
absorption, an initiative, forcing and preferential absorption, is faster and more complete. The process of absorption does not need consuming the energy of the body, so that it cannot increase the burden on the gastrointestinal function, in which active polypeptides can play a role of carrier for the transport. Along with the rapid development of molecular biology, biochemistry and modern protein engineering technology, the research on bioactive peptides has made an epoch-making progress and is highly valued by the researchers.

Cordyceps militaris polypeptide is a hydrolysate prepared directly from Cordyceps militaris by enzymolysis solution technology, and then concentrating and drying, with very strong physiological activities and functional diversity. Physiological and pharmacological activities of Cordyceps militaris polypeptide are mainly to activate related enzyme systems, promote intermediary metabolisms or control the DNA transcription and translation, simultaneously activate the reticuloendothelial system and macrophage, promote the transformation of lymphocytes, and as a nonspecific immune enhancer and regulator, activate the immune competent cells, especially lymphocytes, lymphokines, mononuclear macrophage system and NK cells, thereby attacking the target cells to play an antitumor role, and also exerting its anti-aging, anticoagulant, hypolipidemic to enhance the immunity, improve the liver function and delay the aging $[3,4]$. In this paper, the immunity improvement, anticancer, antioxidation and antimicrobial activities of active polypeptides from Cordyceps militaris are reviewed, in order to provide a solid theoretical help for the further development and application of active polypeptides of Cordyceps militaris.

\section{Brief introduction of polypeptides}

As early as in the 19th century, Engels had proposed that protein is a way of life. Polypeptides are active small molecule substances between amino acids and proteins. In addition to the characteristic functions of proteins, they also have their own unique functions, such as easiness to be digested and absorbed, hypotensive effect, lowering blood lipid and cholesterol, antimicrobial activities, antioxidation, and improvement of mineral absorption. As is known to all, peptide is a biological active substance involved in the function of many kinds of cells and an indispensable participant involved in various complex biological activities in the body.

Bioactive peptides are specific protein fragments that have a positive effect on the function and state of the body and may ultimately affect the health of the body. They are the important chemical messengers between cells and organs, and can regulate the life processes, such as growth, development, reproduction, and metabolism. Bioactive polypeptides have shown several highlights, that is, they can be directly absorbed faster and more completely without the need to be digested through a process of initiative, forcing and preferential absorption, in which the process of absorption does not need consuming the energy of the body, so that it cannot increase the burden on the gastrointestinal function, and can play a role of carrier for the transport. With the rapid development of molecular biology, biochemistry and modern protein engineering technology, the research on bioactive peptides has made an epoch-making progress and is highly valued by the researchers. At present, hundreds of different kinds of biological active peptides that exhibit a variety of biological functions have been found in organisms, such as immune active peptide and hypolipidemic peptides, anti-aging peptides and so on. Studies have found that they can also be used as drugs, vaccines, diagnostic reagents and enzymes mainly for the application in medicine, health care product, food and cosmetic industry.

\section{Cordyceps militaris polypeptide}

Cordyceps militaris polypeptide is a composition of drug gene proteins and nucleosides, contains six compound with cyclic dipeptides, and can coordinate and balance the cardiovascular, nervous, endocrine, visceral, blood, metabolism, and immune systems. Cordyceps militaris polypeptide can slow the heart rate and improve the tolerance to hypoxia, thereby protecting the cardiovascular system; can inhibit a variety of pathogenic bacteria, and has a certain anti-inflammatory, antitussive and 
antiasthmatic effects; has an inhibitory effect on the nervous system, playing a sedative and analgesic effect. Zhu Zhenyuan et al [5] separated and purified a acidic peptide with analgesic effect from the mycelia of Cordyceps gunnii by the method of physical and chemical analysis combined with biological detection method. In addition, Cordyceps militaris polypeptide can also improve the body's immunity and produce an anti-tumor effect. Some experts believe that Cordyceps militaris polypeptide will be developed into a new, efficient, broad-spectrum and safe functional peptide with anti-tumor activity. Qian Kangnan Professor et al [6] prepared a Cordyceps polypeptide that is a water-soluble mixture obtained by refining Cordyceps protein powder containing greater than $60 \%$ $80 \%$ of high-quality Cordyceps protein with the specific enzymolysis engineering technology after the separation and degreasing with super critical $\mathrm{CO}_{2}$ extraction and fractionation, using Cordyceps militaris and Cordyceps sinensis as the raw materials. The material-yield ratio of Cordyceps polypeptides prepared in this way was $3-5: 1$, which can be said to be a "miraculous polypeptide" with a price higher than gold. Experiments have demonstrated that due to the high content of active ingredients in Cordyceps, coupled with the unique advantages of polypeptides, such as small molecules, Cordyceps polypeptide can play a role in $\mathrm{Mg}$ levels. The results from the safety evaluation in the pharmacology and functional detection have also verified that the Cordyceps polypeptide has exhibited no toxicity and side effect, but a higher nutritional value and bioavaibility.

\section{Applications of cordyceps polypeptides}

\subsection{Improvement of Cordyceps polypeptide on the immunity}

Immunoreactive peptides include immune enhancing peptides and immunosuppressive peptides. Immune enhancing peptides can enhance the immune function of the body and exert a nonspecific immunoenhancement on immunodeficiencies and tumors, such as thymic peptides that are separated from thymus or serum system and can induce and stimulate immature lymphocytes, while immunosuppressive peptides are mainly used for the prevention of transplantation rejection and the treatment of autoimmune diseases, such as cyclosporine separated from the fungal culture liquid. Pharmacodynamic experiments have indicated that Cordyceps militaris polypeptide can significantly improve multiple immune indexes in animal experiments, promote the transformation of lymphocytes and improve the cellular immune function of the body; eliminate superoxide free radicals in the body and protect the stability and permeability of cell membranes; significantly improve the activity of NK cells. NK cells are the non-specific part in the cellular immunity, can play their roles directly as a killer cell, and have a good anti-tumor effect, indicating that they are one of the most important components in the cellular immunity.

Studies by Jin Lili at Liaoning University showed that Cordyceps militaris polypeptide could enhance the body immune function through multiple aspects affecting the immune system, in which a Cordyceps militaris polypeptide hydrolysate obtained directly from Cordyceps militaris by enzymolysis technology were given orally to mice successively for 30 days, and the results indicated that the Cordyceps militaris polypeptide hydrolysate could significantly improve the number of antibody producing cells and the level of serum hemolysin, improve the carbon clearance function and the phagocytic capacity of monocyte-macrophage, enhance the delayed allergy, and increase the activity of NK cells in mice. Thus, Cordyceps militaris polypeptide can enhance the immune function of mice.

\subsection{Inhibition of Cordyceps militaris polypeptide on tumors}

Malignant tumors are one of the main diseases threatening human survival, the curative effect of single-drug therapy on them is poor, and the damage caused by the radiotherapy and chemotherapy on the immune system of the host is severe. The screening of effective anti-tumor drugs from natural medicines has become an important way for developing antitumor drugs throughout the world. 
Cordyceps militaris should be an ideal substitute for the traditional Cordyceps, and more and more scholars have paid attention $\mathrm{n}$ to its medicinal value. Professor Li Can [7] at Shandong Traditional Chinese Medicine University found that Cordyceps militaris polysaccharide could significantly inhibit esophageal cancer, breast cancer and gastric cancer cells, but not significantly inhibit lung adenocarcinoma and colon cancer cells; Cordyceps militaris polypeptide could significantly esophageal cancer, breast cancer, gastric cancer and lung adenocarcinoma cells, but not significantly inhibit colon cancer cells.

\subsection{Antibacterial effects of Cordyceps militaris polypeptide}

In recent years, due to the abuse of antibiotics, drug-resistant strains in clinic have been on the increase, resulting in rise in the mortality of clinical infectious diseases. The medical community has been working to find efficient and safe antimicrobial drugs. As is known to all, there are many active components with low toxicities in traditional Chinese medicines, so that to search for antibacterial resources from natural products has become a hot topic. Antibacterial peptides and antiviral polypeptide are widely distributed in nature, and can found in both prokaryotic and eukaryotic organisms, such as cyclic peptides, glycopeptides and lipid peptide obtained from bacteria and fungi. Park et al [8] separated and purified a protein CMP from fruiting bodies, Jack et al. [9] obtained a polypeptide Cordymin from the fermentation broth of Cordyceps militaris etc., and both had a certain degree of inhibitory effect on the different fungi and bacteria; Gao et al at Guizhou University [10] found that they also presented different degrees of inhibitory effects on bacteria and fungi, which were consistent with the related reports of some researchers, indicating that these substances can be well used in biological and medical fields.

\subsection{Antioxidant activities of Cordyceps militaris polypeptide}

A part of free radicals produced in the metabolism process of the body serves as the protection of the body by eliminating pathogenic microorganisms, and the other part is to maintain the balance of the body through complex changes in order to maintain the homeostasis of the internal environment. When the body's homeostasis does not remain, the free radicals generated in the body will not be cleared away in time and accumulated to a certain extent, which will pose a threat to human health, leading to the occurrence of certain diseases. Therefore, the research on the antioxidants that can eliminate the redundant free radicals appears particularly important. Shen Qiying [11] found that Cordyceps militaris polypeptide showed an obvious scavenging effect on oxygen free radicals and hydroxyl radicals, its scavenging hydroxyl radical was stronger than that of mannitol, a specific hydroxyl radical scavenger, and Cordyceps militaris extraction liquid exhibited a dose-dependent scavenging effect on both oxygen free radicals and hydroxyl free radicals.

\section{Conclusions}

As a species of medicinal fungi, Cordyceps militaris has shown an excellent medical and nourishing effect, are widely used in food, medicine, healthcare and other industries now, and has now become a topic that attracted many research scholars' attention. However, the reports on the effective components of Cordyceps militaris mainly focus on the research of Cordycepic acid, Cordycepin and Cordyceps polysaccharides, while those on the protein polypeptide are not enough, and the cost of Cordyceps polypeptide obtained by a specific enzymolysis biotechnology is very expensive. Therefore, this paper sets out the application of Cordyceps militaris in four aspects, such as its immunity improvement, anticancer, antioxidation, and antimicrobial effect, in order to lay the foundation and provide the necessary basis for the further research on small-molecule bioactive peptides in the fruiting body of Cordyceps militaris. 


\section{References}

1. J.Y. Song ch., B.K. Yang, K.S. Ra, J.M Sung. J Microbiol Biotechnol. 8, 536-9(1998).

2. T.B. Nag wh. J Pharm Pharmacol. 57, 1509-19(2005).

3. S.Y. Won pe. Journal of Ethnopharmacology. 96, 555-61(2005).

4. R.M. Yu yw, L.Y. Song, C.Y. Yan, Z. Zhang, Y. Zhao. Cabohydrate Polymers. 70, 430-6(2007).

5. Z.Y. Zhu, X. Chen, J.P. Zhou, A.J. Liu, Y.J. Zhang, Y.M. Zhang. Food Science. 30, 200203(2009).

6. K.G. Qian. China fungal industry science and Technology Conference, (2005).

7. C. Li, B. Yan, F. Sun, L. Wang. Shandong Journal of Traditional Chinese Medicine. 32, 744746(2013).

8. B.T. Park, K.H. Na, E.C. Jung. Korean Journal Physiology Pharmacology. 13, 49-54 (2009).

9. H.W. Jack, B.N. Tzi, H.X. Wang. Phytomedicine. 18, 387-392(2011).

10. Y.Y. Gao, L.H. Zhou, Z.Y. Pan, H.F. Liao. Guangdong Agricultural Sciences. 11, 183-185(2013)

11. Q.Y. Shen. Research Cordyceps oxygen free radical and hydroxyl free radical action. Guangxi Plant. 3, 252(2001). 